Nephrologe 2021 · 16:26-32

https://doi.org/10.1007/s11560-020-00470-2

Angenommen: 17. November 2020

Online publiziert: 11. Dezember 2020

(c) Springer Medizin Verlag GmbH, ein Teil von

Springer Nature 2020

\section{Redaktion}

C. Erley, Berlin

J. Floege, Aachen

W. Kleophas, Hamburg

\title{
Oliver Gross
}

Klinik für Nephrologie und Rheumatologie, Universitätsmedizin Göttingen, Göttingen, Deutschland

\section{COVID-19 und die Niere - Klinik}

\section{Wie die Nephrologie die klinische und intensivmedizinische Versorgung der Patienten fundamental verbessert}

teidigen und früher präventive Maßnahmen ergreifen zu können. Bis vor Kurzem war das Virus gänzlich unbekannt, seine Virulenz ist beängstigend. Daher schaffen wissenschaftliche Erkenntnisse über bekannte Pathomechanismen Vertrauen und Zuversicht in unsere medizinischen Abwehrmöglichkeiten. Kurz gesagt, die COVID-19 Forschung weltweit macht Mut, auch wenn insbesondere die Qualität der klinischen Forschung unter dem Zeitdruck und einem gewaltigen Bias an verschiedenen medizinischen Systemen leidet. Die Datenqualität aus Deutschland erscheint potenziell höher als in manch anderen Ländern, gerade weil uns mit einer gesamtgesellschaftlichen Kraftanstrengung die Kontrolle über das Krankheitsgeschehen bisher nicht entglitten ist. Auch deshalb prägt derzeit Deutschland die weltweite grundlagenwissenschaftliche, internistische und intensivmedizinische COVID19-Forschung ganz wesentlich mit. Diese internationale Sichtbarkeit bei COVID19 durch Spitzenforschung aus Deutschland gilt insbesondere auch für die $\mathrm{Ne}$ phrologie. Deren Nutzen und Bedeutung soll im Nachfolgenden dargestellt werden.

\section{Das klinische Problem: Spitzenmedizin bei limitierten Personalressourcen?}

Machen wir eine kurze Zeitreise zurück in den März 2020. Die Situation in Italien machte deutlich, dass das erfahrene medizinische Personal und die Arbeitsmoral die knappsten Ressourcen darstellen [18]. Da Krankenhäuser selbst schnell Hotspots werden können, bildeten in Deutschland unsere Hausärzte (und Gesundheitsämter) an vorderster Front einen weltweit herausragenden Schutzwall für unsere Krankenhäuser. Dennoch ist es auch in Deutschland eine Utopie zu glauben, in den Krankenhäusern hätten v.a. Internisten und Anästhesisten mit entsprechend internistisch geprägtem Pflegepersonal mit Begeisterung die Versorgung von COVID19-Patienten übernommen. Ein buntes Volk von „Freiwilligen“ meldete sich, zumeist jüngere Ärzte, vom Unfallchirurgen bis zum Augenarzt, und Pflegepersonal aus allen Bereichen. Wirkliche Spezialisten standen zumeist eher in der zweiten Reihe als Konsiliarius oder für Notfallsituationen zur Verfügung. Entsprechend wichtig war und ist die interdisziplinäre, vertrauensvolle $\mathrm{Zu}$ sammenarbeit aller Fachbereiche, um Kollateralschäden durch menschliches Versagen, Überforderung oder Personalmangel $\mathrm{zu}$ vermeiden. Aus der Not geboren, aber eigentlich eine ernüchternde, ganz wertvolle Erkenntnis: Ein gemeinsames Miteinander im Gesundheitssystem mit allen Fachdisziplinen macht viel mehr Spaß und funktioniert viel besser. Meiner Meinung haben in Deutschland dieses Zusammenrücken und Wir-Gefühl die Morbidität und Letalität ganz deutlich gesenkt.

In Sitzungen von Krisenteams wurde schnell klar, welche Fachrichtung in welchem Bereich von COVID-19 seine jeweiligen Stärken hat. Dies zählt aber , an der Front“ der eigentlichen Krankenver- 
Hier steht eine Anzeige.

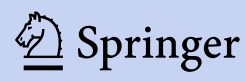




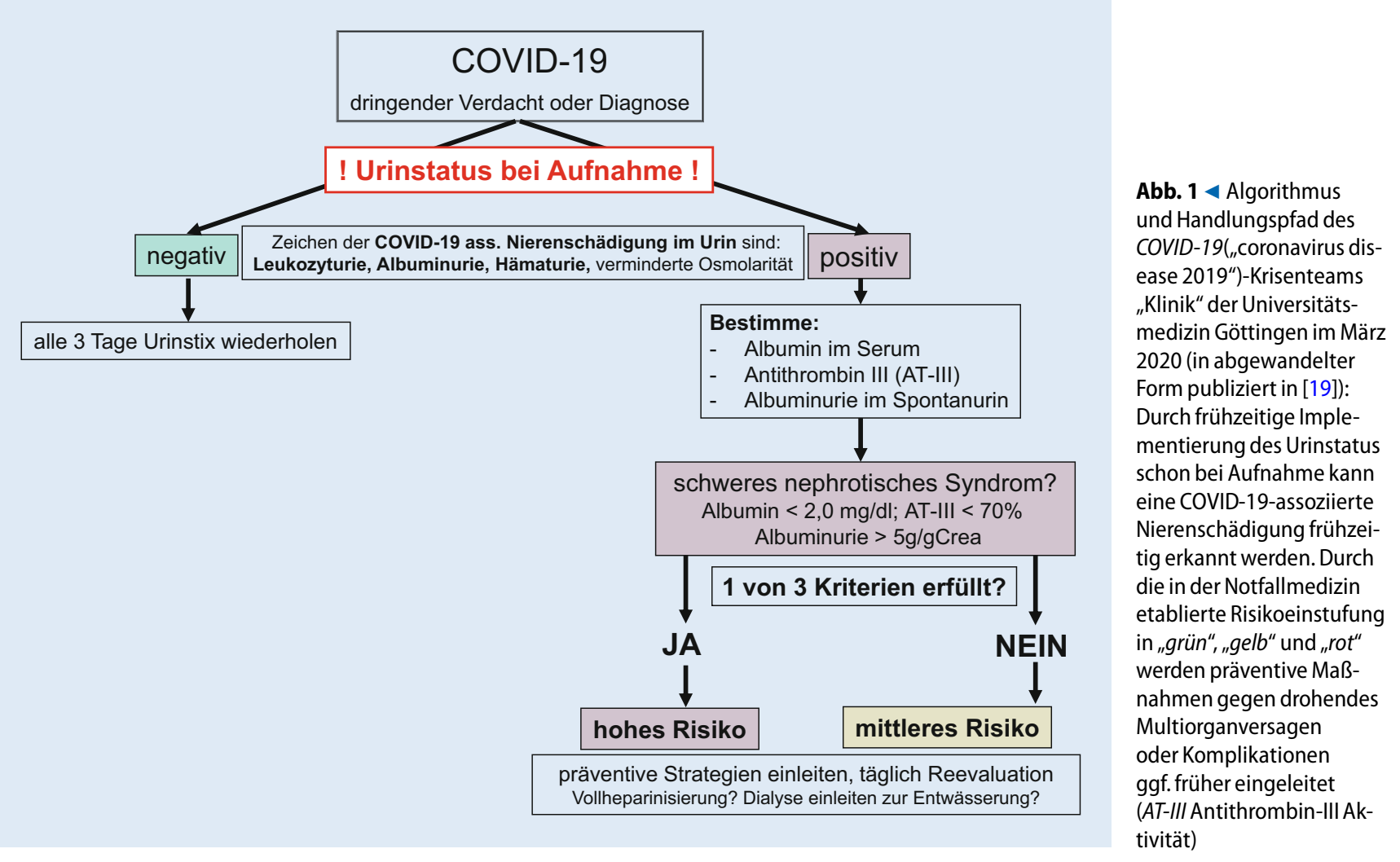

sorgung wenig: Den Sieg, also die Genesung des kritisch kranken COVID-19Patienten, erlangt man mit einer Teamleistung, die nur so stark sein kann wie die Schwächsten im Team. Entsprechend muss jeder im Team seine Stärken nutzen, um die Schwächen anderer mit einfachen Handlungspfaden zu verbessern.

\section{》) Genesung erlangt man mit einer Teamleistung, die nur so stark sein kann wie die Schwächsten im Team}

Wie eingangs beschrieben, prägt die Nephrologie die weltweite COVID-19Forschung ganz wesentlich mit. Damit fällt Ihnen eine sehr schöne Aufgabe zu: Sie können mit Ihrer Beratung und mit einfachen Empfehlungen die Bedeutung Nephrologie bei der Versorgung von COVID-19-Patienten verdeutlichen und so die Teamleistung Ihres Krankenhauses entscheidend verbessern:

\section{Patienten-Surveillance: die Niere als Seismograph des Multiorganbefalls}

Gehen wir in der Zeit wieder zurück in den März 2020. Sie fühlen sich nun durch das bisher Gelesene nephrologisch gewappnet für den ersten COVID-19-Patienten? Nun, leider nein, denn bereits der erste schwere COVID-19-Patient in Göttingen verwies uns durch seine Komplexität medizinisch und wissenschaftlich deutlich in die Schranken. Wieso konnte ein sportlicher Mann mittleren Alters so rasch in ein Multiorganversagen gelangen? Die Vielzahl unerklärlicher Befunde führte aber auch zu offenen multidisziplinären Diskussionen und Hypothesenentstehung: Das Muster der Proteinurie wies auf glomeruläre (und tubuläre) Schäden hin. Das niedrige Serumalbumin von weniger als 2,0 g/dl und eine Antithrombin(AT)-III-Aktivität von weniger als $70 \%$ werteten wir als weitere Zeichen für eine Nierenbeteiligung bei COVID-19. Unsere Kollegen in KölnMerheim konnten diese Hypothese mittels Urinmikroelektrophorese untermau- ern. Dies führte noch im März $2020 \mathrm{zu}$ wichtigen Konsequenzen:

1. Bei Neuaufnahmen in der Universitätsmedizin Göttingen wurde schon bei Aufnahme des Patienten ein Handlungspfad zur Früherkennung von Multiorganbeteiligung und drohenden Komplikationen mittels Urinstatus etabliert (• Abb. 1). Sehr hilfreich war hier die Leiterin der Notaufnahme der Universitätsmedizin Göttingen, Frau Prof. S. Blaschke, die als Nephrologin leicht vom Wert einer Urinanalyse zu überzeugen war.

2. Zur Validierung unserer Hypothese starteten wir eine multizentrische Kohortenstudie in Kooperation mit Aachen, Köln-Merheim und Hamburg. Im Sinne der ICH(International Conference on Harmonisation of Technical Requirements for Registration of Pharmaceuticals for Human Use)-GCP(Good Clinical Practice)Qualität einer klinischen Studie in Zeiten einer Pandemie wurde das Studienprotokoll bei clinicaltrials.gov (No. NCT04347824) als weltweit erste nierenassoziierte COVID-19-Studie angemeldet, durch die Ethikkom- 
mission geprüft und der statistische Analyseplan erstellt.

3. Unsere "Nierenhypothese" wurde von uns als Algorithmus in einer Pilotstudie in The Lancet publiziert [19].

4. Unsere Göttinger Hypothese wurde durch das Hamburger Team um Prof. T. B. Huber in herausragender Form grundlagenwissenschaftlich untermauert $[3,20]$.

Die beiden Arbeiten aus Hamburg mit den Titeln „Multiorgan and renal tropism of SARS-CoV-2" und "SARSCoV-2 renal tropism associates with acute kidney injury" sind nephrologische Spitzenforschung, die man im (kurzen) Original gelesen haben sollte [3, 20]. An der Post-mortem-Kohorte konnte erstmals belegt werden, dass SARS-CoV-2 die Niere befällt. Der Nierenbefall bei SARS-CoV-2 ist zudem - auch wenn es sich um Post-mortemUntersuchungen handelt - mit Nierenschaden, Erkrankungsschwere und Letalität assoziiert. Für den „bodenständigen "Nephrologen sind die Befunde aus Hamburg wissenschaftlich begeisternd, aber angesichts der Aussagekraft einer Urinproteinanalyse mit glomerulären und tubulären Schaden keine Überraschung. Überraschend ist für mich der Gegenwind einiger Publikationen (aus dem Journal of the American Society of Nephrology), die die Nierenschäden allein durch schwere Sepsis bei COVID19-Patienten zu erklären versuchen. Natürlich liegt die Wahrheit in der Mitte: Je nach individuellem Patienten, seinen Komorbiditäten und seinem Krankheitsverlauf wird die Nierenschädigung mehr durch den SARS-CoV-2-Befall der Niere bedingt oder mehr durch das Kreislaufversagen und die Sepsis. Die Nephrologie tut sich mit einem rein akademischen Wettstreit „Nierenbefall durch COVID19 versus sekundärer Nierenschaden“ keinen Gefallen. Die pragmatische Lösung im Sinne der besseren Versorgung der Patienten ist, dass natürlich beide Möglichkeiten für den Nierenschaden verantwortlich sind - wie es uns im Übrigen ja schon die banale Urinanalyse lehrt.

Nephrologe 2021 · 16:26-32 https://doi.org/10.1007/s11560-020-00470-2

(c) Springer Medizin Verlag GmbH, ein Teil von Springer Nature 2020

\section{Gross}

COVID-19 und die Niere - Klinik. Wie die Nephrologie die klinische und intensivmedizinische Versorgung der Patienten fundamental verbessert

\section{Zusammenfassung}

Dieser Artikel soll den klinischen Nutzen des wachsenden Wissens über die Angriffswege von SARS-CoV-2 (,severe acute respiratory syndrome coronavirus $2^{\prime \prime}$ ) aufzeigen: Neben der Lunge kann SARS-CoV-2 multiple Zelltypen in anderen Organen wie z. B. in den Nieren befallen und sich dort replizieren. Wichtige Schädigungswege des Virus wie Endothelitis der Gefäße, thrombotische Ereignisse und Zytokinausschüttung sind noch unvollständig verstanden. COVID-19 ("coronavirus disease 2019") ist eine intensivmedizinische, aber v.a. eine internistische Systemerkrankung, bei der alle internistischen Disziplinen gefordert sind. Unter diesen findet insbesondere die Nephrologie auf verschiedenen Wegen Zugang zum Kampf gegen COVID-19: Die Urinuntersuchung kann Hinweise auf Multiorganbefall, Endothelitis, Mikrothromben, Mikrozirkulationsschäden usw. erbringen. Erfahrungen mit niedrigem Serumalbumin und Antithrombin-III-Aktivität bei nephrotischen Patienten helfen, um andere Fachdisziplinen auf die nachlassende Wirkung von Schleifendiuretika und Heparin hinzuweisen. Das nephrologische Wissen um Komplikationen von Hypoalbuminämie und Diuretika- „Resistenz" muss bei COVID19-Intensivpatienten mit erhöhtem extrazellulären Lungenwasser dazu führen, Nierenersatzverfahren frühzeitiger einzusetzen, um Intubationen noch verhindern zu können. Nutzen Sie die Niere als Seismograph für schwere Verläufe bei COVID-19 und bringen Sie Ihr nephrologisches Wissen für die Optimierung der intensivmedizinischen Versorgung ein. Beides zusammen hat das Potenzial, die Morbidität und Letalität erheblich weiter zu senken.

\section{Schlüsselwörter}

Coronavirus · Nephrologie · Intensivstationen . Risikostratifizierung · Präventive Maßnahmen

\section{COVID-19 and the kidneys-Clinical aspects. How nephrology contributes to fundamentally improve care of patients}

\section{Abstract}

The aim of this article is to explain the clinical benefits of the growing knowledge about severe acute respiratory syndrome coronavirus 2 (SARS-CoV-2). In addition to the lungs, SARS-CoV-2 can invade multiple cell types in other organs, such as the kidneys and replicate there. Important damaging pathways of the virus, such as vascular endotheliitis, thrombotic events and systemic cytokine release are still incompletely understood. Coronavirus disease 2019 (COVID-19) is a systemic disease that necessitates intensive medical care and in particular, internal medicine involvement and represents a major challenge for all disciplines of internal medicine. Among these, nephrology in particular is involved in the fight against COVID-19 in a variety of ways: urine investigations can provide indications of multiple organ involvement, endotheliitis, microthrombi and microcirculation damage, etc. Experience with low serum albumin levels and antithrombin III activity in nephrotic patients helps to point out the decreasing effects of loop diuretics and heparin to other specialist disciplines. Nephrological knowledge of the complications of hypoalbuminemia and "resistance" to diuretics must lead to an early implementation of renal replacement procedures in order to be able to prevent mechanical ventilation in COVID19 intensive care patients with increased extracellular lung fluid. The kidneys can be used as a seismograph for severe courses of COVID-19 and nephrological knowledge can be brought to use to optimize the intensive medical care for critically ill patients. Both together have the potential to considerably reduce morbidity and mortality further.

\section{Keywords}

Coronavirus · Nephrology · Intensive care units · Risk stratification · Preventive measures 


\section{Infobox 1 "Faktencheck" der Universitätsmedizin Göttingen für die "Nichtnephrologen" der COVID-19-Behandlungsteams von März 2020}

Die Handlungsanweisungen und präventiven Maßnahmen werden kurz erklärt, um die Anwendbarkeit für Nichtnephrologen zu erleichtern und die Zielparameter wie "Vollheparinisierung" oder "Volumenentzug" früher zu erreichen.

Wichtigste Fakten zum "capillary leak"/ nephrotischen Syndrom, verursacht durch COVID-19: „capillary leak" und Nierenbeteiligung

- bei schwerem AT-III-Mangel: Patient vollheparinisieren (oder auf andere Antikoagulanzien ausweichen; Achtung: höhere Heparindosis erforderlich)

Wieso? Heparin wirkt über AT-III; AT-III-Mangel verursacht Thromben, und Heparin wirkt nicht richtig!

- zunehmende Dyspnoe/Ödeme/ extravaskuläres Lungenwasser: Minusbilanz anstreben

20-40 mg Furosemid i.v., bis $1000 \mathrm{mg} / \mathrm{Tag}$ (hohe Dosen nötig; z. B. $250 \mathrm{mg}$ Furosemid im Perfusor über 6-8h mit $5 \%$ Humanalbumin aufziehen); erwäge frühzeitige Dialyse zum Wasserentzug Wieso? Albuminverlust erniedrigt onkotischen Druck, führt zu schweren interstitiellen Ödemen; Lasix ${ }^{\circledR}$ bindet an Albumin, wirkt von luminaler Seite bei Hypalbuminämie nicht richtig (braucht höhere Dosen)

- frühe antibiotische Therapie

Wieso? Schwere Immuninkompetenz durch (renalen) Immunglobulinverlust

- Medikamentenspiegel bestimmen bei kritischen Medikamenten

Wieso? Gestörte Plasmaeiweißbindung der Medikamente führt zu anderen Spiegeln und verkürzt die Wirkdauer

Wie lassen sich die Veränderungen im Urin, Serumalbumin und AT-III, erklären?

Mögliche Zeichen eines direkten virusbedingten inflammatorischen tubulären, glomerulären, endothelialen oder interstitiellen Schadens der Niere spiegeln sich im Urinstatus in Form von niedriger Urinosmolarität, Albuminurie, Hämaturie und Leukozyturie wider. Zudem weist der Urinstatus bei einem Kreislauf- und Multiorganversagen einer schweren systemischen COVID-19 auf das akute Nierenversagen hin [21]. Aus rein pragmatischen Gründen fasst unser Algorithmus diese beiden Schädigungsmechanismen (direkter Virusbefall der Nieren und sekundäre Nierenschädigung durch Multiorganversagen) als „COVID-19-assoziierter Nierenschaden " zusammen. Entsprechend können die Veränderungen im Urin, insbesondere die Proteinurie, nicht nur als Indikator der Schwere der Erkrankung dienen, sondern selbst wesentlich die Erkrankungsschwere beeinflussen.

\section{I) Urinveränderungen können als Indikator für die Erkrankungs- schwere dienen, diese aber auch stark beeinflussen}

Die Rationale der beiden zusätzlichen Parameter „Serumalbumin“ und „ATIII" ergibt sich aus dem deutlich gestiegenen Verbrauch an Humanalbumin und AT-III in Krankenhäusern während der ersten COVID-19-Welle und aus unserer Pilotstudie, bei der unsere kränksten Patienten die niedrigsten Werte aufwiesen [19]. Serumalbumin und AT-III sind wegen ihrer Rolle in der Pathogenese der Sepsis Routineparameter in der Notfallund Intensivmedizin [22], aber natürlich auch tägliche Routine in der Nephrologie bei nephrotischen Patienten mit renalem Verlust dieser Substanzen durch einen geschädigten glomerulären Filter [23]. Mir sind keine Daten bekannt, dass COVID-19 zu einem schweren renalen Verlust von Serumalbumin und ATIII führt. Die niedrigen Werte im Blut scheinen eine Kombination aus „Kapillarleck“, gesteigertem renalen Verlust, gesteigertem Verbrauch und verminderter hepatischer Produktion zu sein, dies ist allerdings nur eine Hypothese. Keine Hypothese sind die (durch niedrige ATIII-Aktivität bedingte) scheinbare „Heparinresistenz" zahlreicher stationärer Patienten und die durch Obduktionen belegten plötzlichen Todesfälle durch Lungenembolien im ambulanten Bereich bei zuvor oligosymptomatischen Patienten, die vielleicht durch eine bessere Surveillance und Prävention thrombem- bolischer Ereignisse hätten verhindert werden können [24-27].

\section{Präventive Maßnahmen: nephrologische Expertise als Schlüssel zur Verhinderung schwerer Krankheitsverläufe}

Das niedrige Serumalbumin erhöht das Risiko für Volumenüberladung, Lungenödem mit erhöhtem extravaskulären Lungenwasser und Kreislaufversagen deutlich, alle diese Komplikationen sind beim COVID-19-bedingten Multiorganversagen als wesentliche Todesursachen beschrieben [3-9]. Die niedrige AT-IIIAktivität triggert nicht nur thrombembolische Ereignisse, sondern hebelt die antithrombotische Wirkung von Heparin aus. Hypoalbuminämie und niedrige ATIII-Aktivität sind nephrologisches Basiswissen, das im Szenario eines neu zusammengewürfelten COVID-19-Teams sehr wertvoll wird. Daher haben wir zu unserer Handlungsanweisung einen „Faktencheck“ (•Infobox 1) mit kurzen, prägnanten Erklärungen beigefügt. Durch die geänderte Plasmaeiweißbindung wurden bei den wichtigsten Medikamenten die Wirkspiegel überprüft, die Dialyse zum Wasserentzug zum Verhindern des Lungenversagens rationaler und früher eingesetzt, Patienten früher und aggressiver antikoaguliert sowie PTT(partielle Thromboplastinzeit)-Zielwerte schneller erreicht. Gerne erinnere mich an die (fast nephrologisch anmutende) Freude eines jungen Chirurgen, dass er selbst nun doch bei seinem Patienten die Minusbilanz mit einer höheren Furosemiddosis erzielt hatte, und an den Eifer einer Augenärztin, nun mit einer deutlich erhöhten Heparindosis doch in den PTT-Zielbereich zu kommen (oder ggf. auf andere Antikoagulantien umzusteigen). Mittlerweile ist der Chirurg nun wieder Chirurg und die Augenärztin wieder Augenärztin, aber die Teamarbeit und der Exkurs in die Innere Medizin und die Nephrologie haben allen Spaß gemacht, die Patientenversorgung wesentlich verbessert und uns besser auf die zweite Welle vorbereitet. 


\section{Ausblick}

Die Validierung unseres Algorithmus im Rahmen der Kohortenstudie wurde vor wenigen Wochen als Abstract auf dem diesjährigen Nephrologenkongress vorgestellt und befindet sich zurzeit im sehr kompetitiven Review-Verfahren. An dieser Stelle können wir der Veröffentlichung unserer Studie nicht vorgreifen: Das Prädiktionsmodell soll es ermöglichen, das Multiorganversagen früher zu erkennen und die präventiven Maßnahmen bereits bei Krankenhausaufnahme zu optimieren. Der strategische Vorteil des Prädiktionsmodells wird überdeutlich, wenn es gelingt, drohende Komplikationen wie Thrombembolien und Volumenüberladung zu verhindern oder Therapien zielgerichteter einzusetzen [28-35]. Konkrete Studien zur Surveillance von COVID-19-Patienten und Pflegepersonal mittels Urinstatus bei Ausbrüchen in Altenheimen sind in der fortgeschrittenen Planungsphase.

\section{Fazit für die Praxis}

- In der aktuellen S2k-Leitlinie „Empfehlungen zur stationären Therapie von Patienten mit Covid$19^{\prime \prime}$ vom 23.11.2020 heißt es in EMPFEHLUNG 6: Bei nachgewiesener Covid-19-Infektion und Notwendigkeit einer Hospitalisierung sollte eine Urinuntersuchung (ggf. wiederholt) mit Bestimmung von Albuminurie, Hämaturie und Leukozyturie erfolgen.

- Neben der Lunge, kann SARS-CoV-2 („,severe acute respiratory syndrome coronavirus $2^{\prime \prime}$ ) auch multiple Zelltypen in anderen Organen wie den Nieren befallen und sich dort replizieren.

- COVID-19 („„Coronavirus disease 2019“) ist eine intensivmedizinische, aber auch eine internistische Systemerkrankung, bei der alle internistischen Disziplinen gefordert sind, auch und insbesondere die Nephrologie.

- Der Nierenbefall bei SARS-CoV-2 ist in Post-mortem-Untersuchungen mit Nierenschäden, Erkrankungsschwere und Letalität assoziiert.
- In einer hypothesengenerierenden Pilotstudie der Universitätsmedizin Göttingen wurde schon bei Krankenhausaufnahme von COVID-19-Patienten ein Handlungspfad zur Früherkennung von Multiorganbeteiligung und drohenden Komplikationen mittels Urinstatus, Serumalbumin und Antithrombin(AT)-III-Aktivität etabliert.

- Nutzen Sie die Niere als Seismograph für schwere Verläufe bei COVID-19, und bringen Sie Ihr nephrologisches Wissen für die Optimierung der intensivmedizinischen Versorgung ein. Beides hat das Potenzial, die Morbidität und die Letalität erheblich weiter zu senken.

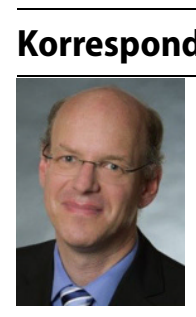
Klinik für Nephrologie und Rheumatologie, Universitätsmedizin Göttingen Robert-Koch Str. 40, 37075 Göttingen Deutschland gross.oliver@ med.uni-goettingen.de

\section{Einhaltung ethischer Richtlinien}

Interessenkonflikt. O. Gross gibt an, dass kein Interessenkonflikt besteht

Für diesen Beitrag wurden von den Autoren keine Studien an Menschen oder Tieren durchgeführt. Für die aufgeführten Studien gelten die jeweils dort angegebenen ethischen Richtlinien.

\section{Literatur}

1. Zhu N, Zhang D, Wang W et al (2020) A novel coronavirus from patients with pneumonia in China, 2019. N Engl J Med 382:727-733

2. Chen N, Zhou M, Dong Xet al (2020) Epidemiolog ical and clinical characteristics of 99 cases of 2019 novel coronavirus pneumonia in Wuhan, China: a descriptive study. Lancet 395:507-513

3. Puelles VG, Lütgehetmann M, Lindenmeyer MT et al (2020) Multiorgan and renal tropism of SARScoV-2. NEngl J Med 383:590-592

4. Mehta P, McAuley DF, Brown M, Sanchez E, Tattersall RS, Manson JJ (2020) COVID-19: consider cytokine storm syndromes and immunosuppression. Lancet 395:1033-1034

5. Ackermann M, Verleden SE, Kuehnel M et al (2020) Pulmonary vascular endothelialitis, thrombosis, and angiogenesis in Covid-19. N Engl J Med 383:120-128

6. Liu PP, Blet A, Smyth D, Li H (2020) The science underlying COVID-19: implications for the cardiovascular system. Circulation 142:68-78

7. Helms J, Tacquard C, SeveracF et al (2020) High risk of thrombosis in patients with severe SARS-CoV-2 infection: a multicenter prospective cohort study. Intensive Care Med 46:1089-1098

8. Varga Z, Flammer AJ, Steiger P et al (2020) Endothelial cell infection and endotheliitis in COVID-19. Lancet 395:1417-1418

9. Pei G, Zhang Z, Peng J et al (2020) Renal involvement and early prognosis in patients with COVID-19 pneumonia. J Am Soc Nephrol 31:1157-1165

10. Emanuel EJ, Persad G, Upshur R et al (2020) Fair allocation of scarce medical resources in the time of Covid-19. N Engl J Med 382:2049-2055

11. Richardson S, Hirsch JS, Narasimhan $M$ et al (2020) Presenting characteristics, comorbidities, and outcomes among 5700 patients hospitalized with COVID-19 in the New York City area. JAMA 323:2052-2059

12. Zhou F, Yu T, Du R et al (2020) Clinical course and risk factors for mortality of adult inpatients with COVID-19 in Wuhan, China: a retrospective cohort study. Lancet 395:1054-1062

13. Huang C, Wang Y, Li X et al (2020) Clinical features of patients infected with 2019 novel coronavirus in Wuhan, China. Lancet 395:497-506

14. Wu Z, McGoogan JM (2020) Characteristics of and important lessons from the coronavirus disease 2019 (COVID-19) outbreak in China: summary of a report of 72314 cases from the Chinese Center for Disease Control and Prevention. JAMA. https://doi. org/10.1001/jama.2020.2648

15. Goyal P, Choi JJ, Pinheiro LC et al (2020) Clinical characteristics of Covid-19 in New York City. N Engl JMed 382:2372-2374

16. Guan W, Ni Z, Hu Y et al (2020) Clinical characteristics of coronavirus disease 2019 in China. N Engl J Med 382:1708-1720

17. Wu C, Chen X, Cai Y et al (2020) Risk factors associated with acute respiratory distress syndrome and death in patients with coronavirus disease 2019 pneumonia in Wuhan, China. JAMA Intern Med 180(7):1-11

18. Fagiuoli S, Lorini FL, Remuzzi G, Covid-19 Bergamo Hospital Crisis Unit (2020) Adaptations and lessons in the province of Bergamo. N Engl J Med 382:e71

19. Gross O, Moerer O, Weber M et al (2020) COVID19-associated nephritis: early warning for disease severity and complications? Lancet 395:e87-e88

20. Braun F, Lütgehetmann M, Pfefferle S et al (2020) SARS-CoV-2 renal tropism associates with acute kidney injury. Lancet. https://doi.org/10.1016/ S0140-6736(20)31759-1

21. Batlle D, Soler MJ, Sparks MA et al (2020) Acute kidney injury in COVID-19: emerging evidence of a distinct pathophysiology. J Am Soc Nephrol 31:1380-1383

22. Sungurlu S, Kuppy J, Balk RA (2020) Role of antithrombin III and tissue factor pathway in the pathogenesis of sepsis. Crit Care Clin 36:255-265

23. Noone DG, lijima K, Parekh R (2018) Idiopathic nephrotic syndrome in children. Lancet 392:61-74

24. Wynants L, Van Calster B, Collins GS et al (2020) Prediction models for diagnosis and prognosis of covid-19 infection: systematic review and critical appraisal. Version 2. BMJ 369:m1328. https://doi. org/10.1136/bmj.m1328

25. Sun Q, Qiu H, Huang M, Yang Y (2020) Lower mortality of COVID-19 by early recognition and 
intervention: experience from Jiangsu Province. Ann Intensive Care 10:33

26. Edler C, Schröder AS, Aepfelbacher M et al (2020) Dying with SARS-CoV-2 infection-an autopsy study of the first consecutive 80 cases in Hamburg, Germany. Int J Legal Med 134:1275-1284

27. Tang N, Bai H, Chen X, Gong J, Li D, Sun Z (2020) Anticoagulant treatment is associated with decreased mortality in severe coronavirus disease 2019 patients with coagulopathy. J Thromb Haemost 18:1094-1099

28. KaragiannidisC,MostertC,HentschkerC, VoshaarT, Malzahn Jet al (2020) Case characteristics, resource use, and outcomes of 10.021 patients with COVID-19 admitted to 920 German hospitals: an observational study. Lancet Respir Med 8(9):853-862

29. Vijayan A, Humphreys BD (2020) SARS-CoV-2 in the kidney: bystander or culprit? Nat Rev Nephrol $14: 1-2$

30. Seymour CW (2020) A cooperation of the doves. Intensive Care Med 18:1-2

31. Poston JT, Patel BK, Davis AM (2020) Management of critically ill adults with COVID-19. JAMA. https:// doi.org/10.1001/jama.2020.4914

32. Cao B, Wang Y, Wen D et al (2020) A trial of lopinavir-ritonavir in adults hospitalized with severe Covid-19. N Engl J Med 382:1787-1799

33. Wang Y, Zhang D, Guanhua D et al (2020) Remdesivir in adults with severe Covid-19: a randomized, double-blind, placebo-controlled, multicentre trial. Lancet 395:1569-1578

34. RECOVERY Collaborative Group, Horby P, Lim WS, Emberson JR et al (2020) Dexamethasone in hospitalized patients with Covid-19-preliminary report. N Engl J Med. https://doi.org/10.1056/ NEJMoa2021436

35. Duan K, Liu B, Li C et al (2020) Effectiveness of convalescent plasma therapy in severe COVID19 patients. Proc Natl Acad SciU SA 117:9490-9496

\section{Hübler, Matthias (Hrsg.) Komplikationen in der Inten- sivmedizin}

\author{
Fallbeispiele - Analyse - Präven- \\ tion
}

Heidelberg: Springer-Verlag 2019, 412 S., 93 Abb., (ISBN: 978-3-66258307-4), Softcover 44,99 EUR

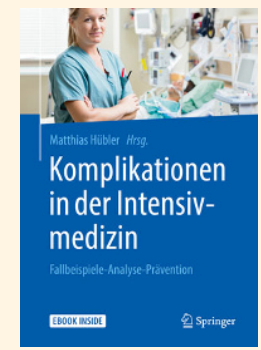

Nach ihrem Erfolg mit "Komplikationen in der Anästhesie" haben Matthias Hübler und sein Autor:innen-Team aus Dresden nun einen Nachfolger mit dem Titel „Komplikationen in der Intensivmedizin" herausgebracht. Die Rezeptur ist unverändert: In 24 Fällen wird die Welt der Intensivmedizin aufgeblättert, alle 24 direkt aus dem wirklichen Leben gegriffen. Es gibt Ärzte und Ärztinnen wie Du und ich mit ihren Stärken und Schwächen, Pfleger und Pflegerinnen wie wir sie schätzen - überwiegend mehr, selten weniger -, und es gibt Patienten und Patientinnen mit ganz normalen, häufigen Erkrankungen

- Intensivmedizin eben. Auf einmal aber passiert etwas Unerwartetes, dann noch etwas manchmal eine ganze verhängnisvolle Kette. Und immer war es nicht einfach nur Schicksal, sondern die handelnden Personen waren Teil des Unheils: Sie haben etwas übersehen, nicht richtig gedeutet, falsch reagiert, sind einer technischen Tücke erlegen oder in eine organisatorische Falle getappt. Auch hier wird nichts konstruiert, sondern alles ist wiederum aus dem wirklichen Leben gegriffen. Matthias Hübler schöpft als langjähriger Intensivmediziner und Protagonist des Critical Incident Reporting System (CIRS) aus einem schier unerschöpflichen Vorrat von kleineren und größeren Fehlern, die sich alle genau in dieser Weise wirklich schon einmal ereignet haben - und zwar nicht etwa selten, sondern als wiederkehrender Teil des intensivmedizinischen Alltags. Und er kennt die Ursachen, die so vielfältig sind wie die Fehler selbst. Oft genug sind psychologische Mechanismen eine treibende Kraft. Mit jedem Fall, den man liest, wird klarer: Dies sind nicht die Fehler der anderen, sondern die Fehler von Dir und mir, von uns allen.
Umso bemerkenswerter ist die konsequente Haltung, mit der das Buch dem Alltagsfaktor Fehler begegnet. Nicht der erhobene Zeigefinger führt durchs Thema, an keiner Stelle wird moralisiert. Ausgehend von der schlichten Feststellung, dass Fehler vorkommen, werden sie benannt, wird ihr Mechanismus analysiert, und werden Hinweise gegeben, wie sie in Zukunft vermieden werden können Die Fälle folgen immer demselben Aufbau. Im ersten Teil wird die Geschichte erzählt und mit fachlichen Informationen zu Krankheiten, Medikamenten und Prozeduren angereichert. Im zweiten Teil der Darstellung geht es dann eher systematisch um die Fehler, die Teil der Geschichte waren. Der Stil ist locker und unterhaltsam, ein kleiner Hauch des guten alten "House of God" weht herein, Manches dabei ist vielleicht etwas verspielt. Die Sachlichkeit der Darstellung und der Ernst des Anliegens gehen aber nie verloren. Nach weniger als einer halben Stunde ist man durch mit einem Fall. Man wurde ertappt, verständnisvoll auf den Fehler und seinen Mechanismus aufmerksam gemacht und ist motiviert es in Zukunft besser zu machen. Obendrein hat man fachlich einen kleinen Happen dazugelernt. Auf diese Weise ist das Buch ein guter Reisebegleiter oder das ideale Nachtschränkchen-Utensil, sei es zu Hause oder im Dienst. Am besten aber liegt es auf der Intensivstation an einem für alle zugänglichen Platz - es sage keine:r, dass sich nicht dann und wann doch einmal zwanzig Minuten für eine kleine geistige Erfrischung finden.

Dem Buch ist hoffentlich der verdiente Erfolg beschieden, so dass man bei einer zweiten Auflage die kleinen Fehler beseitigen kann, die sich immer wieder in die Grammatik, und ganz manchmal in - vorwiegend internistische - Inhalte eingeschlichen haben.

Prof. Dr. med. Sebastian M. Schellong, Dresden 